\title{
SIX DEgREES OF SEPARATION TO IMPROVE ROUTING IN OPPORTUNISTIC NETWORKS
}

\author{
Carlos O. Rolim ${ }^{1}$, Valderi R. Q. Leithardt ${ }^{1}$, Anubis G. Rossetto ${ }^{1}$, \\ Tatiana F. M. dos Santos ${ }^{2}$, Adriano M. Souza ${ }^{2}$, Cláudio F. R. Geyer ${ }^{1}$ \\ ${ }^{1}$ Institute of Informatics - Federal University of Rio Grande do Sul (UFRGS) \\ P.O. Box 15.064 - 91.501-970 - Porto Alegre - RS - Brazil \\ \{carlos.oberdan, valderi.quietinho, agmrossetto, geyer\}@inf.ufrgs.br \\ ${ }^{2}$ Postgraduate Program in Production Engineering - Federal University of Santa Maria \\ (UFSM) - 97105-900 - Santa Maria - RS - Brazil \\ taty.nanda@gmail.com, amsouza@ufsm.br
}

\begin{abstract}
Opportunistic Networks are able to exploit social behavior to create connectivity opportunities. This paradigm uses pair-wise contacts for routing messages between nodes. In this context we investigated if the "six degrees of separation" conjecture of small-world networks can be used as a basis to route messages in Opportunistic Networks. We propose a simple approach for routing that outperforms some popular protocols in simulations that are carried out with real world traces using ONE simulator. We conclude that static graph models are not suitable for underlay routing approaches in highly dynamic networks like Opportunistic Networks without taking account of temporal factors such as time, duration and frequency of previous encounters.
\end{abstract}

\section{KEYWORDS}

Opportunistic Networks, Routing Algorithm, Data Dissemination, Complex Networks, Small-World Networks, Graphs

\section{INTRODUCTION}

In everyday life improvements of mobile devices with wireless technologies, such as IEEE 802.11, WiMAX, Bluetooth, and other short range radio solutions (sensor devices), lead to a myriad of new ubiquitous applications ranging from simple messaging systems to cyber-physical environments that may even encompass an entire city [1].

Making this prospect a reality, raises a number of challenges. One of these concerns the use of mobile computing as an underlying technology to supply collaboratively sensed data and social networking metadata for these ubiquitous services. In wide-scale urban scenarios, only using wireless infrastructures (e.g. cellular, WLAN, or WiMAX networks) to provide services is not satisfactory, as it is very unlikely that wireless infrastructures alone will be able to provide enough bandwidth and coverage for the huge number of devices spread throughout the environment [2]. Another important consideration is that current mobile computing applications are infrastructure-centric, which forces users to be acutely aware of their connectivity environment, with many applications only working when the networking infrastructure is available.

An alternative way of overcoming these limitations is the use of Opportunistic Networks. Opportunistic Networks are a recent mobile networking paradigm stemming from research into conventional Mobile Ad Hoc NET-works (MANET) [3]. In this paradigm, nodes are assumed to 
be mobile, and the forwarding of messages is based on the Store-Carry and Forward concept. Opportunistic Networks represent the first attempt to close the gap between human and network behavior by adopting a user-centric approach to networking and exploiting user nodes' mobility as an opportunity—rather than a challenge — to improve data forwarding [4].

A problem in this context is how to route data between nodes in a suitable way (i.e. with high delivery rate, low latency and low overhead). There are several initiatives which can be taken to meet these requirements. The proposed solutions involve adopting different approaches, ranging from "naïve" (i.e. the use of "blindly" flooding techniques to reach the destination node) to "intelligent" (i.e. with an emphasis on social factors in routing messages). However, to the best of our knowledge, none of the existing studies has attempted to apply small-world network concepts [5] for this purpose. The idea of incorporating this class of complex networks in Opportunistic Networks is driven by the most recent studies of human mobility and social contacts; these are using graph models to better understand and represent the degree of social connection between people [6]. Hence, this paper aims to investigate the question of whether the "six degrees of separation" conjecture of small-world networks can be used as a basis for routing improvements in Opportunistic Networks.

In summary, the contributions made by this paper are twofold: first there is a demonstration that static graph models are not suited to underlay routing approaches in highly dynamic networks (like Opportunistic Networks which do not take account of temporal factors such as time, duration and frequency of encounters). Second it shows that the application of these findings in a simple routing algorithm can surpass the performance of other algorithms in terms of the number of messages, delivery and overhead. In addition to being original, these features signal the direction for further research in this area.

The rest of this paper is structured as follows: The next section provides information about Opportunistic Networks and some related initiatives, with a focus on routing; Section III provides a brief outline of small-networks and some concepts used in this work; Section IV describes our study and examines the existence of small-world networks in some selected real traces; Section V analyzes the results of the experiments; and, finally we conclude this paper in Section VI.

\section{ROUTING IN OPPORTUNISTIC NETWORKS}

Opportunistic networking is a technology with good prospects of fulfilling the ubiquitous vision [7]. This new paradigm is sometimes referred to in the literature as delay-tolerant networks (DTN). The DTN architecture and protocols are currently under study and being defined by the Internet Research Task Force's Delay Tolerant Networking Research Group. Opportunistic Networks is a general concept as it does not assume any compatibility with the Internet architecture, nor any a priori knowledge regarding the network topology, areas of disconnection, or future link availability [4]. Opportunistic Networks seeks to simplify the complexity at the network layer by removing the assumption of physical end-to-end connectivity while providing connectivity opportunities for pervasive devices when no direct access to the Internet is available. Pervasive devices, equipped with different wireless networking technologies (e.g. Bluetooth), are frequently out of range from a network but are in the range of other networked devices, and sometimes cross areas where some type of connectivity is available (e.g. Wi-Fi hotspots). Thus, they can opportunistically exploit their mobility and contacts for data delivery [7]. As a result, a new type of DTN has emerged called Pocket Switched Networks (PSN). PSN is a networking paradigm, which uses human mobility and a store-and-forward strategy to solve the communication problem outside the connectivity islands [8]. In this paper we will employ the term Opportunistic Network because it includes more general concepts. 
The current widely used approaches in this area range from special-purpose networks - like the Sámi Network Connectivity project — to general-purpose networking — like the Haggle project. In specific fields, it should be noted that there are ongoing attempts, mainly in the area of data dissemination and routing protocols employing different underlying techniques. There is no consensus among researchers about how to classify these protocols, but the most commonly used criteria for classification are the role of contextual information and the social factors for routing. Thus, protocols shift from being "naïve" (i.e. using no or slight contextual information) to "intelligent" (i.e. using contextual information such as location, history, connectivity or social information for intelligent decision- making). In this way, [9] classified protocols into three classes: context-oblivious, mobility-based, and socially relevant/context-aware. As it is not within the scope of this article, we will not explain and separate it into categories. However the following initiatives should be cited: Epidemic, Spray\&Wait (and the Spray variants like Spray\&Focus, Fuzzy-Spray and others), Prophet, BubbleRap, MobySpace, AFRON, CAR, HiBOp, Propicman and the most recent Prophet improvement called DRAFT. All of these represent attempts to optimize message routing (see [10] for further information).

After conducting an investigation we found that the most recent and influential studies are using some kind of socially-aware factors. This led us to explore in greater depth how the social interactions are represented and analyzed. We found that the two most widely used classes of complex networks for this purpose are scale-free networks and small-world networks. Scale-free networks are networks where degree distribution of links between nodes follows a mathematical function called power law. In contrast, small-world networks are networks that follow the "six degrees of separation" conjecture [5]. The scope of this paper will be confined to small-world networks because it was evident that none of the studies in the literature was concerned with this area and this stimulated us to make further investigations. In the next section we will examine related concepts to provide the reader with a general background of this subject.

\section{SMALL-WORLD NETWORKS}

Complex networks are graphs with non-trivial features like those that occur in simple networks such as lattices or random graphs. Real examples of this graph can be cited like biological, technological and social networks. This scientific area is relatively new and a good deal of work is being carried out using basically two classes of complex networks: scale-free networks and small-world networks. In this paper, we will avoid dealing with the former because we intend to exploit the "hub" features of these networks in future work.

In 1967, the psychologist Stanley Milgram and his colleagues carried out an experiment with 296 individuals in Nebraska and Boston. Everyone was given a letter which had to be sent to a target individual in Massachusetts. When sending this letter each person had to follow the instruction: "If you know the target person, give the letter to him, otherwise give it to someone whom you think is closer to the target." In the course of many trials, 64 letters reached the target, through 5.2 intermediaries [5]. Since this experiment, this amount has become known in popular culture as the "six degrees of separation" and indicates the degree of separation between two nodes. This principle is also often spoken of as small-world phenomenon. Watts \& Strogatz [11] studied networks with low degrees of separation by carrying out a set of rewiring experiments on graphs, and observed that a small world network can be found between a highly ordered network and a randomly connected network just by varying the randomness $p$ of the network connections between regularity $(p=0)$ and disorder $(p=1)$ (Figure 1). 


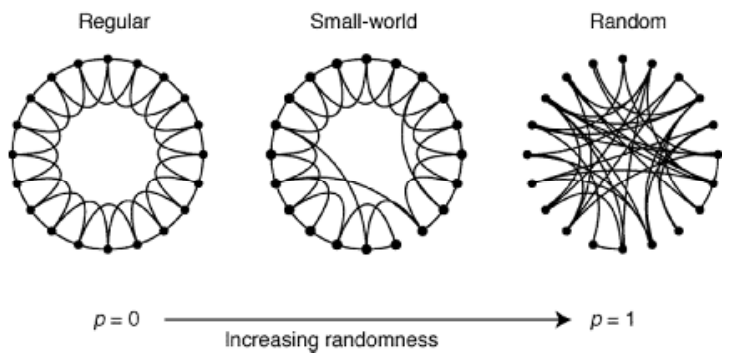

Figure 1. Family of graphs varying randomness $p$ of the network connections [11]

A small-world network is characterized by a clustering coefficient $\mathrm{C}$ and a path length L. Smallworld networks can be represented by directed or undirected graphs. Owing to the "undirected nature" of Opportunistic Networks, we will only examine concepts of small-world networks as undirected graphs (for more information see a good review and tutorial conducted by [12]).

Let $k_{n}$ be the degree (number of neighbors) of node $n$, and $e_{n}$ be the number of edges (connected pairs) among the nodes directly connected to node $n$. In undirected networks, the clustering coefficient $C_{n}$ of node $n$ is defined as $C_{n}=2 e_{n} / k_{n}\left(k_{n}-1\right)$. Since $e_{n}$ is never larger than $k_{n}\left(k_{n}-1\right) / 2$, the clustering coefficient is a

fraction between 0 and 1.If the neighborhood is fully connected (forms a clique), the clustering coefficient is 1 and a value close to 0 means that there are hardly any connections in the neighborhood. The average clustering coefficient (also known as the clustering coefficient of the graph or clustering coefficient for the whole network) is given by Watts and Strogatz as the average of the local clustering coefficients of all the nodes, that is $\check{\mathrm{C}}=\frac{1}{n} \sum_{1}^{N} C_{n}$.

The length of a path is the number of edges forming it. There may be multiple paths connecting two given nodes. The shortest path length, also called distance, between two nodes $n$ and $m$ is denoted by $L_{(n, m)}$. The average shortest path length is a measure of efficiency, that is the average number of stops needed to reach two distant nodes in the graph. It is represented by

$L_{(\mathrm{G})}=1 / n *(n-1) * \sum_{n=1}^{N} \sum_{m=1}^{N} L_{(n, m)}$ [12]. The lower the result, the more efficient is the network in providing ease of circulation. In comparison, the diameter is the maximum length of all possible shortest paths.

According to [11], [13] two properties distinguish a small world network. First, the average clustering coefficient $\mathrm{C}$ is larger than for a corresponding random network with the same number of connections and nodes. The clustering coefficient expected for a regular rectangular grid network is zero while for a random network the probability $p$ of connection of two nodes is the same for neighboring nodes as for distant nodes. Second, the average path length $L$ scales like $\log N$, where $N$ is the number of nodes. For regular (grid) networks $L$ scales as $N d$ where $d$ is the dimension of the space and for random networks $L$ scales as $\log N$. As result, $C$ is large and $L$ is small in a small-world network. In other words, small-world networks have a low path length and high clustering coefficient. These striking features have led us to analyze the application of small-world concepts in Opportunistic Networks as an alternative for routing messages.

\section{SMALL-WORLD CONCEPTS IN OPPORTUNISTIC NETWORKS}

After introducing the small-world networks, we seek to understand how this class of graph can be used in Opportunistic Networks. First we will use some tools in the field of social network analysis to determine if selected real-world scenarios can be characterized as small-world networks. We selected three real mobility traces for our analysis from Dartmouth College's 
CRAWDAD public repository covering different scenarios from sparse (campus) to dense (conference) and collated in different contexts and through different methods. The selected dataset consisted of MIT's Reality, Infocom'06 and SIGCOM'09. These are public and wellknown by the academic community in the area of Opportunistic Networks. All the datasets provide traces representing contacts between nodes. We define a contact as the period of time during which two devices are in mutual radio transmission range and can exchange data. The details of these datasets are listed below:

- Reality: The MIT's Reality Mining trace logged contact communication, proximity, location, and activity information from 100 subjects at MIT over the course of the 2004-2005 academic year. According to the authors, this data represents over 350,000 hours ( 40 years) of continuous data on human behavior. This valuable supply of data on complex social systems has implications for a variety of fields. The data is collected using smartphones by Bluetooth scans with a scanning interval of 5 minutes. We obtained data from between 4,294,800 and 21,276,000 seconds with 97 devices.

- Infocom: This trace contains the contacts between participants in an Infocom'06 workshop. It includes Bluetooth sightings by groups of users carrying Intel motes (iMote), a small battery operated Bluetooth v1.1 radio with approximately 30 meter range for four days at the IEEE Infocom Conference in Grand Hyatt Miami. The devices perform a device discovery every $120 \pm 5$ seconds. In our case, we used data collected between 5,497 and 34,2915 seconds with 77 mobile devices (20-97), plus 21 static devices.

- SIGCOMM: The trace contains data collected by an opportunistic mobile social application called MobiClique. The application was used by 76 people during the SIGCOMM 2009 conference in Barcelona, Spain. The application uses Bluetooth for opportunistic contact discovery and data communications. The device discovery is performed every $120 \pm 10$ seconds. The experimental devices have a Class 2 Bluetooth v2.1 radio with a range of 10 to 20 meters. The data sets include traces of Bluetooth device proximity, opportunistic message creation and dissemination, and the social profiles (friends and interests) of the participants. In our tests we used a proximity trace set that contains all the nearby Bluetooth devices reported by the periodic Bluetooth device discoveries between 21 and 349,811 seconds. We filtered the user_ids below 100 to get only the experimental devices (user_ids >= 100 are external Bluetooth devices seen during the experiment).

We started our analysis by trying to obtain a better understanding of the selected dataset scenarios. By using Gephi software, we visualized and analyzed the network graphs. Table 1 shows the results of our analysis. The graphical representation of contact graphs from each dataset is shown in Figure 2. From the graphical representation we can see

Table 1. Resuls of graph analysis.

\begin{tabular}{l|ccc} 
& Reality & Infocom & SIGCOMM \\
\hline Year & $2004-2005$ & 2006 & 2009 \\
Setting & Campus & Conference & Conference \\
Total nodes & 96 & 98 & 76 \\
Edges & 305 & 4414 & 3730 \\
Total Communities & 4 & 3 & 6 \\
Diameter & 2 & 2 & 2 \\
Radius: & 1 & 1 & 2 \\
Density & 0.677 & 0,929 & 0,731 \\
Average Node Degree & 64,271 & 90,082 & 49,079 \\
Number of Shortest Paths & 9120 & 9506 & 5700 \\
Average Path Length & 1.3241 & 1.071 & 1,269 \\
Average Clustering Coefficient & 0,817 & 0,951 & 0,782
\end{tabular}


communities formed by node contacts and also note that all the scenarios have some common feature like a small diameter and radius, that characterizes a large number of node contacts. In Table 1, attention should be paid to the Average Path Length and Average Clustering Coefficient. As we pointed out earlier, a small-world network has a low path length and high clustering coefficient. In the case of the graphs above, when they were analyzed, they all had a low Average Path Length (rounded down to 1) and a high Average Clustering Coefficient (rounded up to 1) that are characteristics of small-world networks topologies. Hence, we can conclude that smallworld concepts can be applied in these kinds of scenarios.

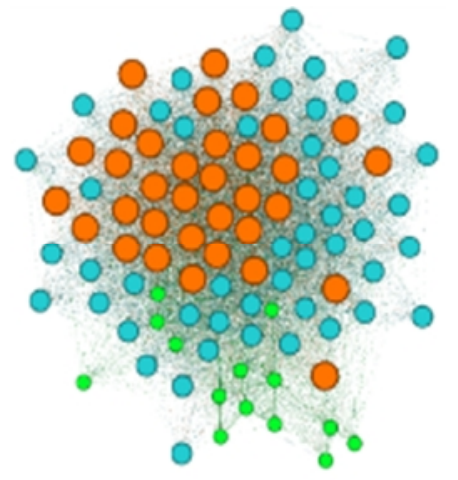

(a)

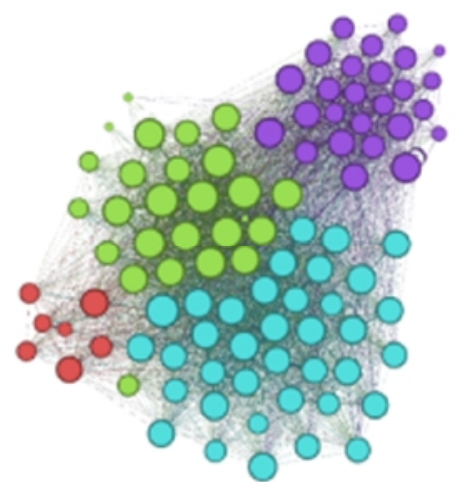

(b)

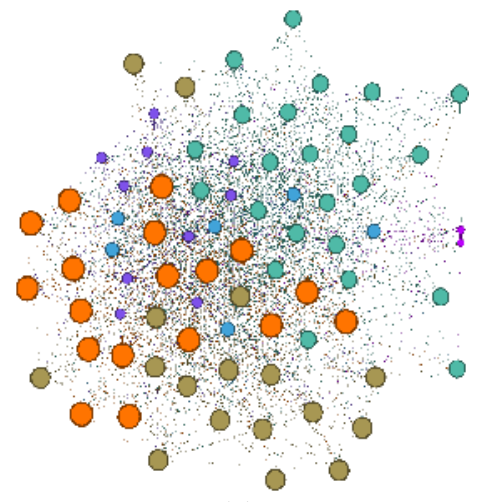

(c)

Figure 2. Diagrams of different datasets (a) Infocom (b) Reality (c) SIGCOMM

\section{SIMULATION AND EXPERIMENTAL RESULTS}

This section will demonstrate how our experiments can apply small-world concepts in the scenarios outlined above for message routing. It starts with a brief description of a simulation setup, and describes some measurement parameters to make inferences about the performance of route protocols. Following this, it shows our attempt to employ small-world concepts for routing and ends with the simulation results.

\subsection{Simulation setup}

Our simulations were carried out by using ONE (Opportunistic Network Environment) Simulator and the trace datasets mentioned earlier, as input. Owing to the different duration length of the collected data (SIGCOMM:349811 seconds $=4.04$ days; Reality: 16981816 seconds $=196.5$ days; Infocom:337418 seconds $=3.9$ days) we adopted the small duration length of three scenarios for simulation time, in all of the experiments: 3.9 days round up $=4$ days $=345600$ seconds. We ran each simulation scenario with the total number of nodes included in each dataset to maintain contact fidelity. We assumed Bluetooth devices with a radio range of 20 meters and transmission speed of $2 \mathrm{Mbit} / \mathrm{s}$. On average, the nodes generated one message per minute (total of 5760 messages) in all of the experiments and the message lifetime was set to 24 hours (1440 seconds). We used message sizes that were uniformly distributed between $100 \mathrm{~KB}$ (text message) and $2 \mathrm{MB}$ (digital photo).

One comment should be made about the duration of the contacts on the traces file: to simulate contacts, ONE requires the timestamps when two nodes enter and leave their mutual radio transmission range. In traces this is represented by the timestamp plus an indication about whether the connection goes up or down. The Reality and Infocom dataset provide this data, but the original SIGCOMM trace only provides the timestamp of the start connection and not when it goes down. Without this information ONE cannot infer the duration of the contact. To overcome 
this, we pre-processed the trace file and inserted missing lines to reflect contacts between nodes (as required by the simulator). We adopted random duration periods ranging from 5 to 30 seconds. In making a comparison of our small-world approach with other works, we selected some current routing algorithms which could be used in simulations. The selected algorithms were Epidemic, Prophet, DRAFT and BubbleRap. These were chosen because they represent the different classes of protocols (as outlined in Section 2) and have been extensively studied by researchers.

Some of the chosen protocols do not need configuration parameters, such as Epidemic. In others, we have to set it to run simulations. For Prophet we set secondsInTimeUnit $=30$. For DRAFT we set familiarThreshold $=120$, degrade $=0.5$ and framesize $=3600$. For Bubblerap we set $K=5$, familiarThreshold $=700$, centralityTimeWindow $=21600$ and epoch_count $=16$ (i.e. simulation time of 345600 seconds / centralityTimeWindow $21600=16$ ).

\subsection{Evaluation metrics}

In the case of evaluation we adopt same performance metrics used by [8] and supported by ONE Simulator:

- Create: Number of messages created during simulation - does not include replicated messages;

- Relayed: number of successful transmissions between nodes;

- Delivered: number of successfully delivered messages;

- Overhead ratio: an assessment of bandwidth efficiency. Uses the formula (Number of Relayed Messages - Number of Delivered messages) / Number of Delivered Messages

- Latency_med: median of average message delay

- Hopcount_avg: Average number of hops between source and destination nodes.

\subsection{Research evolution}

To support the argument that each node is separated by, at most, six hops (six degree conjecture) we formulate a quite simple algorithm called Small-World:

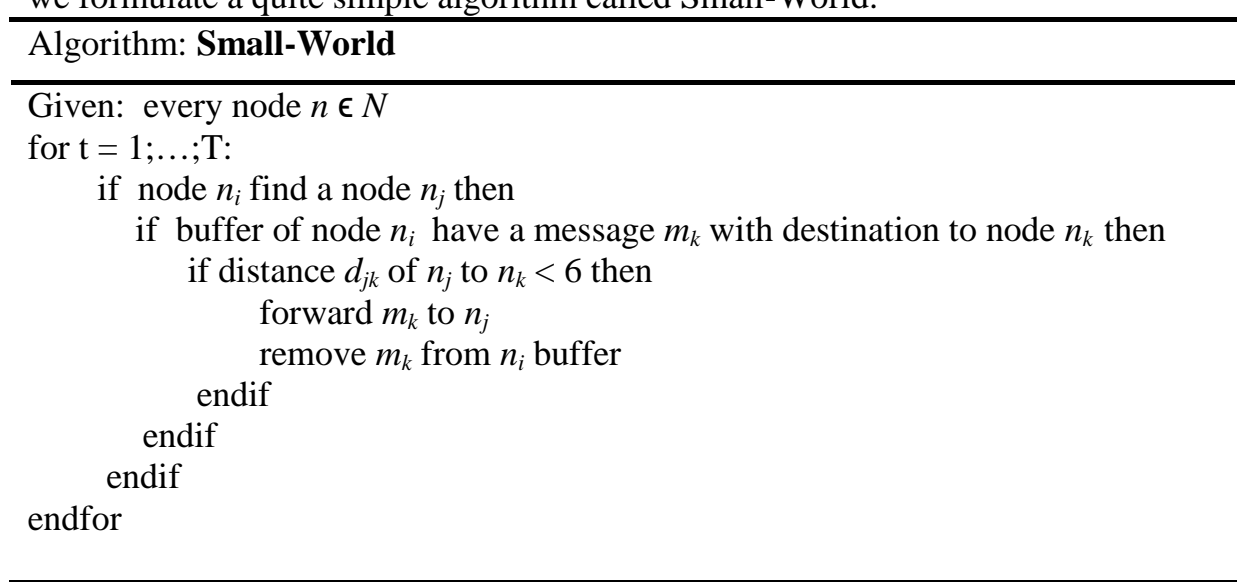

It strictly obeys the small-world assumption and we think it could route messages between the nodes. The simulation results (Table 2) were disappointing. Small-World only delivered $0.45 \%$ to $3.2 \%$ of 5760 messages in all the scenarios. 
International Journal of UbiComp (IJU), Vol.4, No.3, July 2013

Table 2. Simulation results from the simple approach.

\begin{tabular}{|c|c|c|c|c|}
\hline & Infocom & Reality & Sigcomm & \\
\hline \multirow{3}{*}{ Epidemic } & 313 & 17 & 154 & Delivered \\
\hline & 1460382 & 11339 & 124361 & Relayed \\
\hline & 4664,7572 & 666 & 806,539 & Overhead \\
\hline \multirow{3}{*}{ Prophet } & 637 & 36 & 196 & Delivered \\
\hline & 937740 & 20722 & 61082 & Relayed \\
\hline & 1471,1193 & 574,6111 & 310,6429 & Overhead \\
\hline \multirow{3}{*}{ DRAFT } & 439 & 27 & 152 & Delivered \\
\hline & 1002289 & 17747 & 25044 & Relayed \\
\hline & 2282,1185 & 656,2963 & 163,7632 & Overhead \\
\hline \multirow{3}{*}{ BubbleRap } & 469 & 27 & 137 & Delivered \\
\hline & 126135 & 1841 & 20892 & Relayed \\
\hline & 267,9446 & 67,1852 & 151,4964 & Overhead \\
\hline \multirow{3}{*}{ Small-World } & 185 & 26 & 118 & Delivered \\
\hline & 1239121 & 10019 & 78151 & Relayed \\
\hline & 4346,7929 & 384,3462 & 661,2966 & Overhead \\
\hline
\end{tabular}

The poor performance of our first attempt encouraged us to think about how to improve it. Thus, we decided to assign a weight to each graph edge to represent the number of contacts between two nodes. When one node encounters another and there is an edge between them, the edge weight will be incremented by one; else (if there is no edge) it will be created with the initial weight $=1$. In view of this, we changed our first approach so that we could forward a message just as if the weight of the edge was greater than $\kappa$ (we used $\kappa=3$ to represent the fact that these nodes had previously encountered each other three times). We called this modification "Weighted Small-World" and the pseudo-code was as follows:

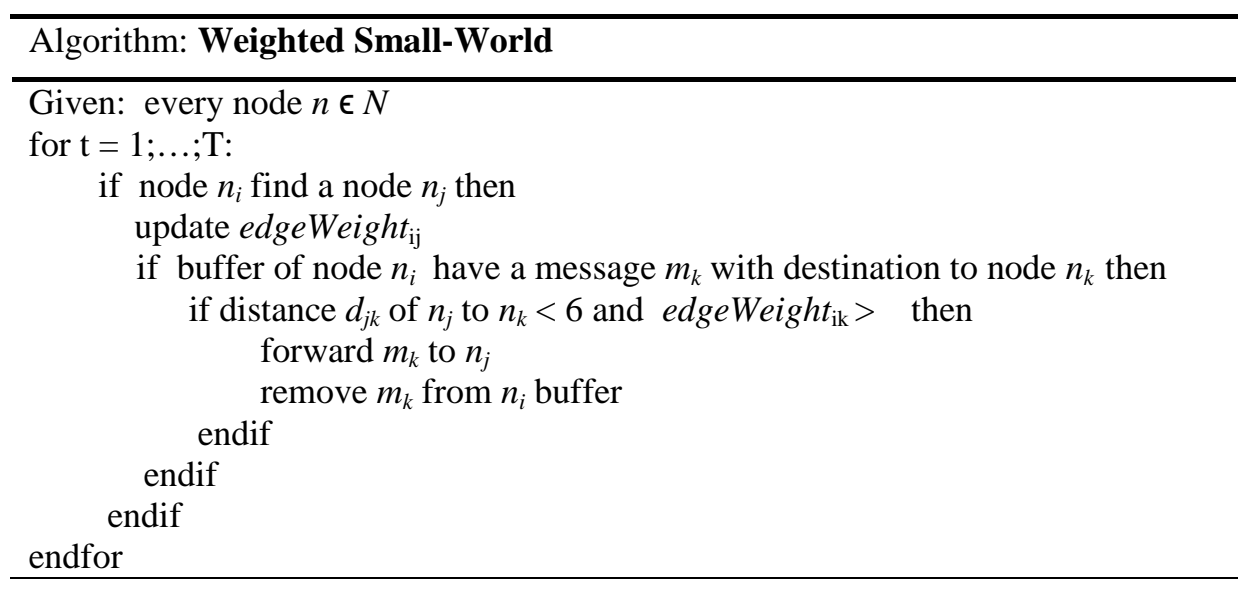

Table 3. Results of the comparison between the two approaches.

\begin{tabular}{|c|c|c|c|c|}
\hline \multirow[b]{2}{*}{ Small-World } & Infocom & Reality & Sigcomm & \\
\hline & $\begin{array}{r}185 \\
4346,7929\end{array}$ & $\begin{array}{r}26 \\
384,3462\end{array}$ & $\begin{array}{r}118 \\
661,2966\end{array}$ & $\begin{array}{l}\text { Delivered } \\
\text { Overhead }\end{array}$ \\
\hline $\begin{array}{l}\text { Weighted } \\
\text { Small-World }\end{array}$ & $\begin{array}{r}299 \\
5014,194\end{array}$ & $\begin{array}{r}29 \\
838,2069\end{array}$ & $\begin{array}{r}126 \\
611,1984\end{array}$ & $\begin{array}{l}\text { Delivered } \\
\text { Overhead }\end{array}$ \\
\hline
\end{tabular}

In Table 3, only the results of Small-World and Weighted Small-World are shown, to make a comparison. We can note a slight improvement in the performance of the weighted version compared with the former, but it is still far from the other protocols used in the simulations. 
After some experiments with simulations, we discovered that used scenarios are highly dynamic and the patterns of the movements of humans lead to vertices in the graph with obsolete edges between them. These edges that was biasing the distance calculation used by our previous SmallWorld approaches, resulting in a poor performance. This occurs because the graph representation does not take account of temporal factors such as time, duration and frequency of previous encounters. A similar assessment of spatio-temporal clusters was previously carried out by [8] and later exploited by the DRAFT protocol [14]. We used this observation to guide our further steps. However, instead of detecting lost spatio-temporal clusters by allowing clusters to decay over time (as used in DRAFT), we formulated an improvement of our previous attempt called Temporal Small World. Our strategy was the same as that used in Weighted Small-World (weighted edges according to number of contacts) but we kept removing the obsolete edges (i.e. the nodes that had not encountered each other in some time interval). The pseudo-code of Temporal Small World can be represented as a variation of Weighted Small-World with some added stages to store the time of contact between two nodes and include a loop to periodically update the edges:

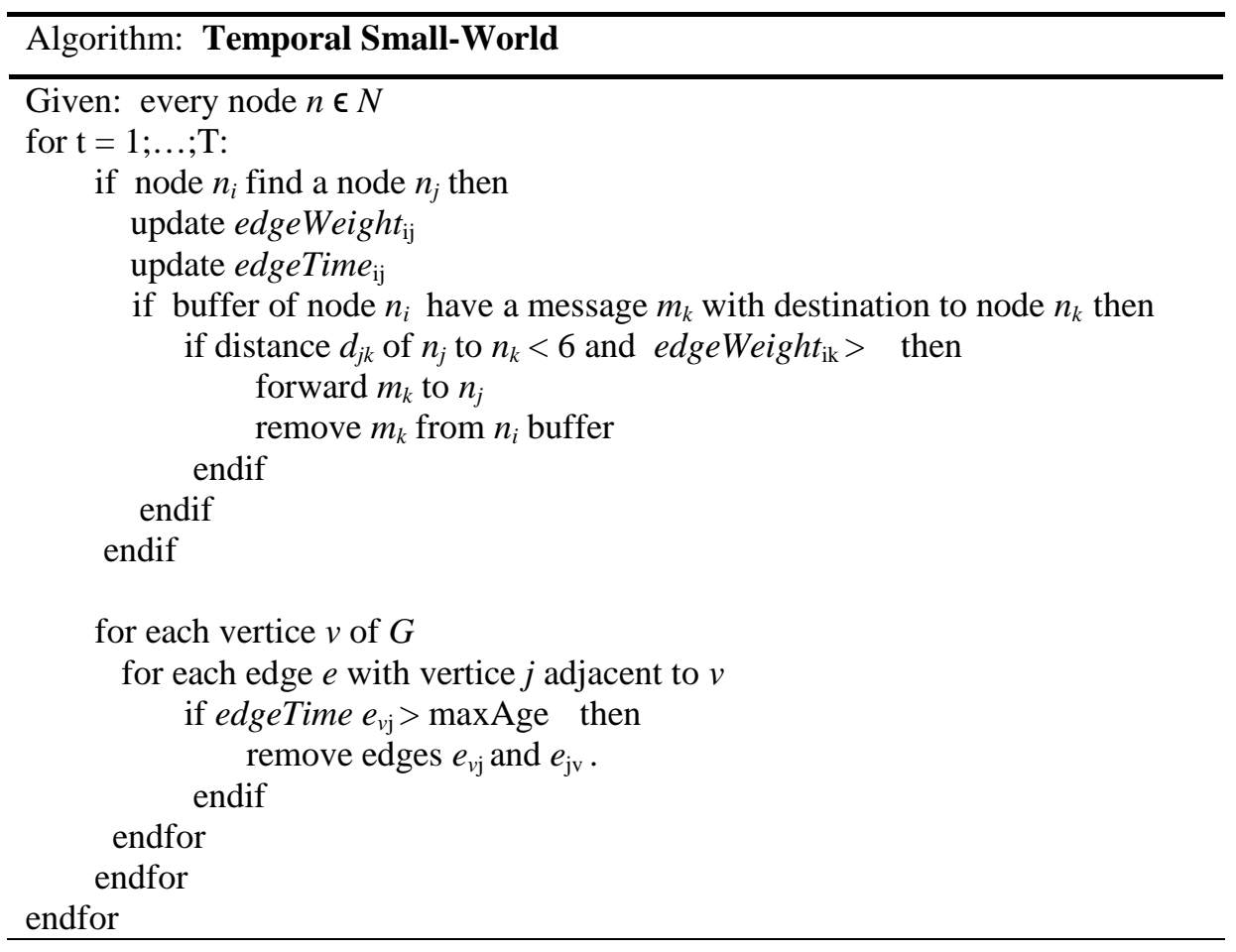

\subsection{Experimental Results}

We conducted a set of experiments to test the performance of our Temporal Small-World proposal. Our first experiment was to understand the implication of value of maxAge $\tau$ in expiration of obsolete edges and how it would have an impact on the routing performance. In the simulations, we tested $\tau$ with values of $1800,3600,21600$ and 86400 seconds (i.e. 30 minutes, 1 hour, 6 hours and 24 hours). The results are shown in Table 4 . 
Table 4. Impact of the variation of $\tau$

\begin{tabular}{|c|c|c|c|c|}
\hline \multirow[t]{2}{*}{$\tau$ (in seconds) } & Infocom & Reality & Sigcomm & \multirow{4}{*}{$\begin{array}{c}\text { Delivered } \\
\text { Relayed } \\
\text { Overhead }\end{array}$} \\
\hline & 486 & 29 & 177 & \\
\hline \multirow[t]{2}{*}{1800} & 787028 & 980 & 27327 & \\
\hline & 1618,3992 & 32,7931 & 153,3898 & \\
\hline \multirow{3}{*}{3600} & 458 & 33 & 166 & \multirow{3}{*}{$\begin{array}{c}\text { Delivered } \\
\text { Relayed } \\
\text { Overhead }\end{array}$} \\
\hline & 1003633 & 6617 & 36395 & \\
\hline & 2190,3384 & 199,5152 & 218,2470 & \\
\hline \multirow{3}{*}{21600} & 363 & 31 & 166 & \multirow{3}{*}{$\begin{array}{c}\text { Delivered } \\
\text { Relayed } \\
\text { Overhead }\end{array}$} \\
\hline & 1354832 & 19467 & 36395 & \\
\hline & 3731,3196 & 626,9677 & 218,2470 & \\
\hline \multirow{3}{*}{84600} & 311 & 33 & 135 & \multirow{3}{*}{$\begin{array}{c}\text { Delivered } \\
\text { Relayed } \\
\text { Overhead }\end{array}$} \\
\hline & 1770544 & 24987 & 74665 & \\
\hline & 5692,0675 & 756,1818 & 552,0741 & \\
\hline
\end{tabular}

As can be seen, the best performance in all of the scenarios was with lower $\tau$ values. This fact indicates our initial belief that obsolete edges really have an impact on message routing. The improvement in performance can be witnessed by the number of messages delivered. In the Infocom scenario, we increased the number of delivered messages from 118 in our first attempt to adopt the original Small-World approach to 486 with Temporal Small-World (+ 24\%) but with $+40 \%$ of overhead.

After this, we carried out some simulations and plotted the performance of Temporal SmallWorld with other selected protocols (Figure 3). It should be highlighted that although it produced $+40 \%$ of overhead, when this criterion was adopted, Temporal Small-World was better than Epidemic and DRAFT in all the scenarios and either worse or better than Prophet and BubleRap, but in all cases with relatively similar results.

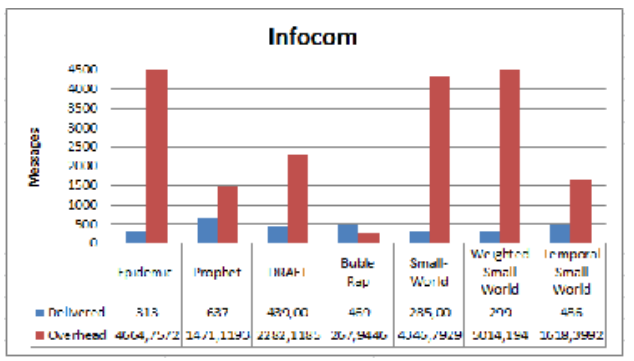

(a) Infocom

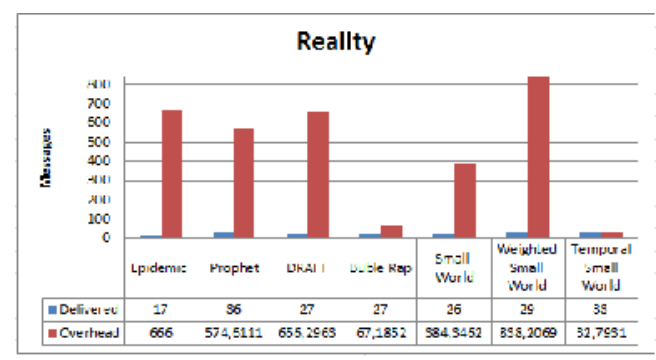

(b) Reality

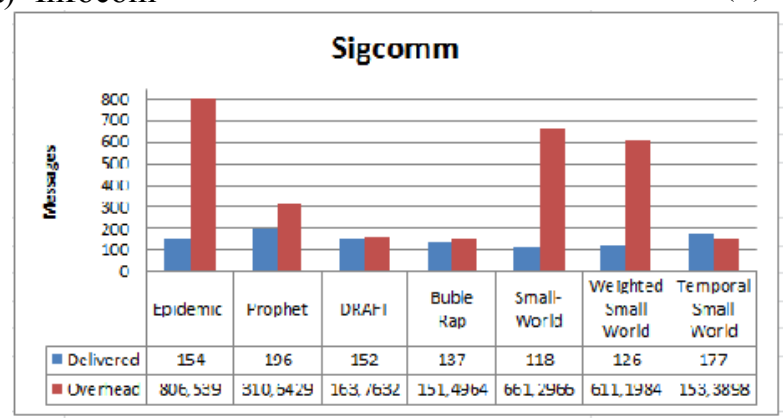

(c) SIGCOMM

Figure 3. Performance of different protocols in selected scenarios: (a)Infocom (b)Reality

(c)SIGCOMM 
With regard to the number of delivered messages, the results demonstrate that Temporal SmallWorld had the second best ratio in all the scenarios (Prophet was the first). What caught our attention was the number of relayed messages of Prophet that ranged from 19\% (Infocom) to 213\% (Reality) and was more than Temporal Small-World in all the scenarios. We recall that to relay messages, mobile devices use battery power for processing and data transmission; thus a high value of this indicator could influence the battery life of these devices. Another finding is the low number of relayed messages of BubbleRap that vary between $24 \%$ to $84 \%$, which is less than Temporal Small-World. We believe that this ratio can be attributed to the strategy of only relaying messages to members of community of interest. However, we argue that this indicator could be better investigated in the light of issues arising from battery and community detection in future research studies.

In the final analysis, we believe that Temporal Small-World can overcome the Prophet results with fine tuning of max age $\tau$ and number of previously encountered $\kappa$ variables. Nevertheless, owing to the wide range of factors involved in human mobility, this task could be better performed by some statistical or machine learning method that was able to predict the most suitable value.

\section{CONCLUSION AND FUtURE WORK}

Opportunistic Networks are able to exploit social behavior to build connectivity opportunities. This study analysed the feasibility of applying the concepts of small-world networks to improve routing.

We proposed a simple approach based on small-world networks called Temporal Small World for routing and showed how it could outperform some popular protocols by means of simulations. It can be concluded that static graph models are not well suited to underlay routing approaches in highly dynamic networks like Opportunistic Networks without taking account of temporal factors such as time, duration and frequency of encounters.

In future work, we will investigate which technique can be used to fine tune max age $\tau$ and the number of previously encountered $\mathrm{k}$ variables. We believe that a prediction of these values using contextual information could improve the performance of our algorithm.

\section{REFERENCES}

[1] S. K. Das, "Pervasive computing vs. cyber-physical systems: A perspective from smart environments," in 2012 IEEE International Conference on Pervasive Computing and Communications Workshops, 2012, pp. 105-105.

[2] M. Conti, S. Giordano, M. May, and A. Passarella, "From opportunistic networks to opportunistic computing," IEEE Communications Magazine, vol. 48, no. 9, pp. 126-139, Sep. 2010.

[3] C. Boldrini, M. Conti, and A. Passarella, "Design and performance evaluation of ContentPlace, a social-aware data dissemination system for opportunistic networks," Computer Networks, vol. 54, no. 4, pp. 589-604, Mar. 2010.

[4] M. Conti and M. Kumar, "Opportunities in Opportunistic Computing," Computer, vol. 43, no. 1, pp. 42-50, Jan. 2010.

[5] S. Milgram and J. Travers, "The Small World Problem - An Experimental Study of the Small World Problem," Psychology Today, vol. 2, no. 4, pp. 60-67, 1967.

[6] D. Karamshuk, C. Boldrini, M. Conti, and A. Passarella, "Human mobility models for opportunistic networks,” IEEE Communications Magazine, vol. 49, no. 12, pp. 157-165, Dec. 2011. 
International Journal of UbiComp (IJU), Vol.4, No.3, July 2013

[7] H. Anh Nguyen and S. Giordano, "Routing in Opportunistic Networks," in Ubiquitous Developments in Ambient Computing and Intelligence, K. Curran, Ed. IGI Global, 2011, pp. 179193.

[8] P. Hui, "People are the network: experimental design and evaluation of social-based forwarding algorithms," Cambridge, 2008.

[9] H. A. Nguyen and S. Giordano, "Context information prediction for social-based routing in opportunistic networks," Ad Hoc Networks, vol. 10, no. 8, pp. 1557-1569, Nov. 2012.

[10] C. Boldrini, J. Y. Le Boudec, A. Chaintreau, and M. Conti, "Deliverable 2.2: Final specification of forwarding paradigms in Haggle," 2008.

[11] D. J. Watts and S. H. Strogatz, "Collective dynamics of 'small-world' networks.," Nature, vol. 393, no. 6684, pp. 440-2, Jun. 1998.

[12] B. J. Prettejohn, M. J. Berryman, and M. D. McDonnell, "Methods for generating complex networks with selected structural properties for simulations: a review and tutorial for neuroscientists.," Frontiers in computational neuroscience, vol. 5, p. 11, Jan. 2011.

[13] D. Simard, L. Nadeau, and H. Kröger, "Fastest learning in small-world neural networks," Physics Letters A, vol. 336, no. 1, pp. 8-15, Feb. 2005.

[14] M. Orlinski and N. Filer, "The rise and fall of spatio-temporal clusters in mobile ad hoc networks," Ad Hoc Networks, Mar. 2013. 